\title{
Ossified Cephalhematoma: How to Differentia it from Intradiploic Hematoma: Case Report
}

\author{
Selçuk Gözcü* and Ahmet Celal iplikçioğlu \\ Department of Neurosurgery, BHT CLINIC Istanbul Tema Hospital, Turkey
}

*Corresponding author: Selçuk Gözcü, Department of Neurosurgery, BHT CLINIC Istanbul Tema Hospital, Atakent Mahallesi 4. Cadde No: 36, Küçükçekmece, 34307, Istanbul, Turkey, Tel: +90-(545)-588-63-36, Fax: +90-(212)-912-43-34

\begin{abstract}
Ossified cephalhematoma is rare and usually occur in newborns due to birth injury. Some ossified cephalhematomas can resorb spontaneously while others require surgery because of cosmetic reasons. Simple excision of new bone is usually sufficient. It should be also differentiated from another clinical entity called intradiploic hematoma which requiers surgery. In reported cases, of infant intradiploic hematoma cases clinical and radiologicial findings are similar to those of ossified cephalhematoma. We believed that the thickness of skull is preserved in the cases of ossified cephalhematoma while thickness of inner and outer tables of skull are significantly decreased in the cases if intradiploic hemotoma.
\end{abstract}

\section{Keywords}

Cephalhematoma, Ossified cephalhematoma, Intradiploic hematoma, Birth injury

\section{Introduction}

Cephalhematoma is the blood collection between the pericranium and skull. It usually occur in parietal region of the newborns, associated with birth injury secondary to use of forceps or vacum device $[1,2]$.

Most of the cephalhematomas resorb spontaneously within 4-6 weeks. However very rarely, displaced periost induce subperiostal osteogenesis resulting in ossified cephalhematoma. Large ossified cephalhemotomas are rare and cause cosmetic deformity requiring surgical correction.

Another patology that should be differentinted from ossifed cephalhemotomasis intradiploic hematoma of the skull [3-5]. Intradiploic hematoma is the expanding bleeding within the diploe between internal and external tables of the skull, following mild head injury. In this report we present a case of large ossified cephalhemotoma with a discussion about differential diagnosis.

\section{Case Report}

A 40-day-old female baby was admitted to the hospital with $7 \times 8 \mathrm{~cm}$ hard left parietal mass. She was delivered vaginally using forceps and the mass was growing since birth. The patient was neurologically intact and blood tests for coagulation disorders were normal.

ACT scan showed left parietal extracranial hypodense mass surrounded with bone density Shell (Figure 1). This apperance is well known as double skull and is charaeteristic for the ossified cephalhemotoma. The contour of the skull is normal. Excision of new bone was planned but the parents of the patient refused the surgery.

\section{Discussion}

Detachment of pericranium (periost) layer due to inward outward movement of the flexible neonatal skull during the trauma causes subperiostal bleeding. Incidence of cephalhemotoma changes between $0.2-2 \%$. In only $3-5 \%$ of them subperiostal osteogenesis occurs and ossified cephal hemotoma developes although most of them resolve spontaneously [1,2].

Histopathologic examination of the new bone shows mature bony structures therefore the term ossification is more appropriate than calcification to describe the process [1].

Ossified cephalhemotoma usually does not cause neurological symptoms hovewer, surgery is indicated for the cosmetic reasons. On the basis of surgical ap-

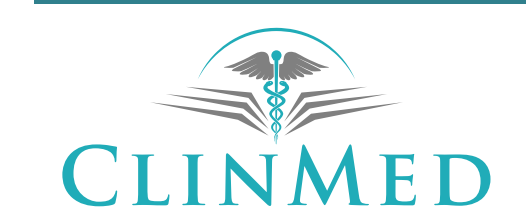

INTERNATIONAL LIBRARY
Citation: Gözcü S, İplikçioğlu AC (2020) Ossified Cephalhematoma: How to Differentia it from Intradiploic Hematoma: Case Report. Neurosurg Cases Rev 3:036. doi.org/10.23937/2643-4474/1710036

Accepted: June 16, 2020; Published: June 18, 2020

Copyright: (C) 2020 Gözcü S, et al. This is an open-access article distributed under the terms of the Creative Commons Attribution License, which permits unrestricted use, distribution, and reproduction in any medium, provided the original author and source are credited. 

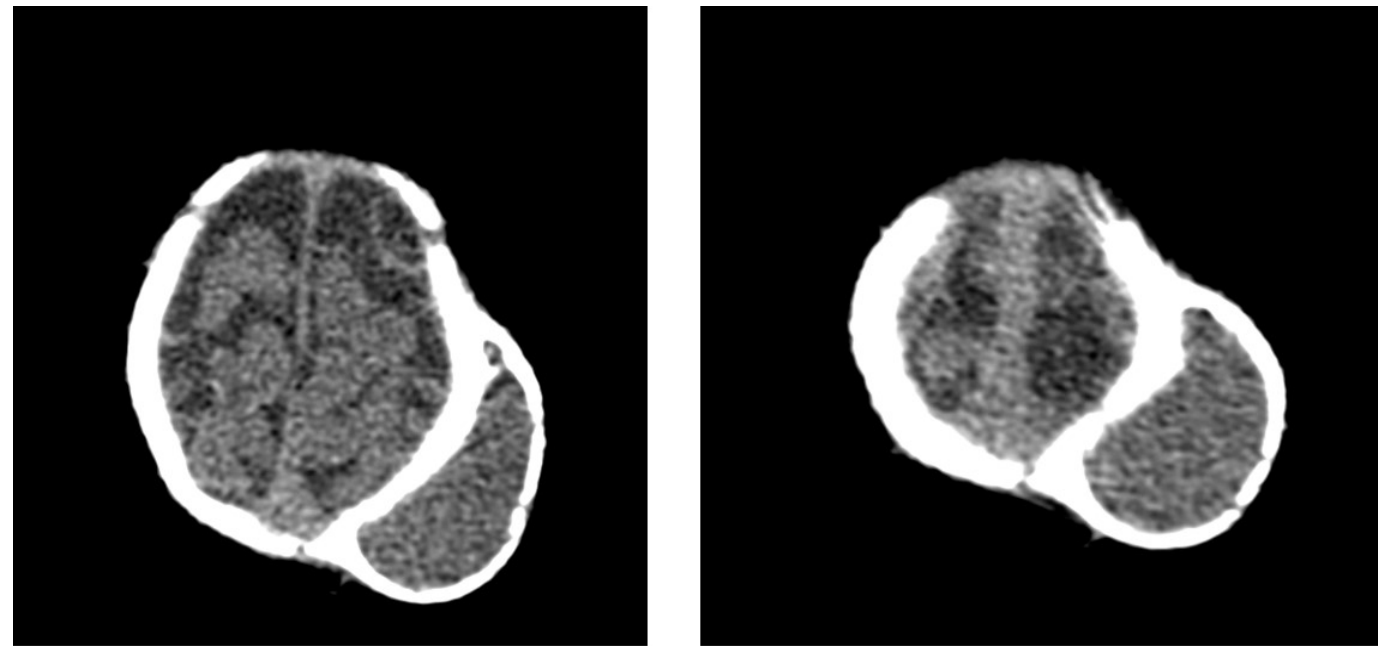

Figure 1: A CT scan shows left parietal extracranial mass with bony shell. A double skull appearance is seen.

proach ossified cephalhematomas is classfied in two types. In type 1 , the contour of the skull is normal while in type 2 depression of the original skull is present [6].

$\mathrm{CT}$ is essential for the diagnosis and excluding the other pathologies. Hovewer, there are some reports describing intradiploic hematomas in newborns and infants with same clinical and radiological findings [35]. We belived that the correct diagnosis may be ossified cephalhematoma in most of these cases. The main confusion about the diagnosis is the misinterpretation of new bone as outer lamella. Some ossified cephalhematomas can resorb spontaneously, therefore it is mandatory to differentiate it from intradiploic hematomas which require surgery. In ossified cephalhematoma cases, the contour and the thickness of the skull under the hematoma is preserved (type 1 ossified cephalhematoma). In type 2 ossified cephalhematoma cases, althoughs light depression of the skull is present, the thickness of skull is still nearly preserved [6]. MRI usually shows the hematoma content as hyperintensity on T1 weighted images and its help is limited in the demonstration of bony structures [2]. Intradiploic hemotomas is rare and usually occur in adults with frontal predilection, secondary to mild injury [7]. Encroachment to intracranial cavity causing neurological deficit and elevated intracranial pressure is common. Thickness of internal and external tables decrease significantly.

Surgical treatment of ossifed cephalhematoma type 1 is the simple excision of the new bone formation. Some authors prefer crainotomy and remodelling the bone in type 2 cases [6]. However in type 2 cases with slight depression of cranium, simple excision of the bone cape is usually sufficent.

\section{Conclusion}

Ossified cephalhematoma is rare and usually occur in newborns due to birth injury. Some ossified cephalhematomas can resorb spontaneously while others require surgery because of cosmetic reasons. It should be also differentiated from another clinical entity called intradiploic hematoma which requiers surgery. Simple excision of the new bone formation is usually sufficent.

\section{References}

1. Liu L, Dong C, Chen L (2016) Surgical treatment of ossified cephalhematoma: A case report and review of the literature. World Neurosurg 96: 614

2. Vigo V, Battaglia DI, Frassantino P, Tamburrini G, Calderelli M (2017) Calsified cephalhematoma as an unusual cause of EEG anomalies: Case report. J Neurosurg Pediatr 19: 46-50.

3. El Damaty A, Elbabaa SK, Unterberg A, Baechli H (2018) Intradiploic skull hematoma in infants: An underestimated diagnosis. J Pediatr Neurol Neurosci 2: 11-16.

4. Luo W, WU M, Chen Q, Zhang B (2018) Intradiploic hematoma in an infant: Case report and literature review. $\mathrm{Br} \mathrm{J}$ Neurosurg 32: 691-693.

5. Yucesoy K, Mertol T, Ozer H (1999) An infantile intraosseous hematoma of the skull. Report of a case and review of the literature. Childs Nerv Syst 15: 69-72.

6. Wong $\mathrm{CH}$, Foo Cl, Seow WT (2006) Calcified cephalhematoma classification, indication for surgery and techniques. $J$ Craniofac Surg 17: 970-979.

7. Tokmak M, Ozek E, Iplikcioglu C (2015) Chronic intradiploic hematomas of the skull without coagulopathy: Report of two cases. Neurocirugia (Astur) 26: 302-306. 